TRANSACTIONS OF THE

AMERICAN MATHEMATICAL SOCIETY

Volume 363, Number 1, January 2011, Pages 521-543

S 0002-9947(2010)05163-3

Article electronically published on August 13, 2010

\title{
PRESENTING THE COHOMOLOGY OF A SCHUBERT VARIETY
}

\author{
VICTOR REINER, ALEXANDER WOO, AND ALEXANDER YONG
}

\begin{abstract}
We extend the short presentation due to [Borel '53] of the cohomology ring of a generalized flag manifold to a relatively short presentation of the cohomology of any of its Schubert varieties. Our result is stated in a root-system uniform manner by introducing the essential set of a Coxeter group element, generalizing and giving a new characterization of [Fulton '92]'s definition for permutations. Further refinements are obtained in type $A$.
\end{abstract}

\section{Contents}

1. Introduction 521

2. Proof of Theorem $1.1 \quad 524$

3. Reducing presentations in $H^{\star}(G / P)$ to those in $H^{\star}(G / B)$

4. Refinements in type $A \quad 531$

4.1. Schubert conditions and bigrassmannians 531

4.2. Bigrassmannians and essential Schubert conditions 533

4.3. Grassmannians and symmetric functions

4.4. A shorter presentation in type $A \quad 536$

4.5. A minimality conjecture 537

4.6. Some symmetric function identities 539

4.7. Proof of Theorem 4.8 540

5. A question 542

Acknowledgements 5542

References $\quad 542$

\section{INTRODUCTION}

The cohomology of the generalized flag manifold $G / B$, for any complex semisimple algebraic group $G$ and Borel subgroup $B$, has a classical presentation due to Borel [B53. Pick a maximal torus $T \subset B \subset G$, choose a field $\mathbb{k}$ of characteristic zero, and let $V=\mathbb{k} \otimes_{\mathbb{Z}} X(T)$, where $X(T)$ is the coweight lattice of $T$. Let $\mathbb{k}[V]:=\operatorname{Sym}\left(V^{\star}\right)$ be the symmetric algebra for $V^{\star}$; it naturally carries an action

Received by the editors November 27, 2008 and, in revised form, June 29, 2009.

2000 Mathematics Subject Classification. Primary 14M15, 14 N15.

Key words and phrases. Schubert calculus, Schubert variety, cohomology presentation, bigrassmannian, essential set.

(C)2010 American Mathematical Society Reverts to public domain 28 years from publication 
of the Weyl group $W:=N_{G}(T) / T$. Furthermore let $\mathbb{k}[V]^{W}$ be the ring of $W$ invariants of $\mathbb{k}[V]$, and $\mathbb{k}[V]_{+}^{W}$ the ideal of $\mathbb{k}[V]$ generated by the $W$-invariants of positive degree. A classical theorem of Borel [B53] states that

$$
H^{*}(G / B, \mathbb{k}) \cong \mathbb{k}[V] /\left(\mathbb{k}[V]_{+}^{W}\right) .
$$

A desirable feature of Borel's presentation is its shortness: since $W$ acts on $V^{\star}$ as a finite reflection group, by Chevalley's Theorem, the $W$-invariants $\mathbb{k}[V]^{W}$ are a polynomial algebra $\mathbb{k}\left[f_{1}, \ldots, f_{n}\right]$, where $n:=\operatorname{dim}_{\mathbb{k}} V$ is the rank of $G$ [H90, Section 3.5]. Hence Borel's presentation shows that $H^{\star}(G / B)$ is a complete intersection, as it has $n$ generators and $n$ relations.

There is a second way to describe $H^{\star}(G / B)$ arising from a cell decomposition of $G / B$. One has

$$
\begin{aligned}
G & =\bigsqcup_{w \in W} B w B & & \text { (Bruhat decomposition), } \\
G / B & =\bigsqcup_{w \in W} X_{w}^{\circ} & & \text { (Schubert cell decomposition), with } \\
X_{w}^{\circ} & :=B w B / B \cong \mathbb{C}^{\ell(w)} & & \text { (open Schubert cell). }
\end{aligned}
$$

Here $\ell(w)$ denotes the Coxeter group length of $w$ under the Coxeter system $(W, S)$, where $S$ are the reflections in the simple roots $\Pi$ among the positive roots $\Phi^{+}$ within the natural root system $\Phi$ associated to our Lie pinning. This gives a $C W$-decomposition for $G / B$ in which all cells have even real dimension. Hence the fundamental homology classes $\left[X_{w}\right]$ of their closures $X_{w}:=\overline{X_{w}^{\circ}}$, the Schubert varieties, form a $\mathbb{Z}$-basis for the integral homology $H_{\star}(G / B)$, and their (Kronecker) duals $\sigma_{w}:=\left[X_{w}\right]^{\star}$ form the dual $\mathbb{Z}$-basis for the integral cohomology $H^{\star}(G / B)$.

The above facts lead to an a priori presentation for $H^{\star}\left(X_{w}\right)$; see also C92, Cor. 4.4] and [GR02, Prop. 2.1]. The Schubert variety $X_{w}$ is the union of the Schubert cells $X_{u}^{\circ}$ for which $u \leq w$ in the (strong) Bruhat order; hence it inherits a cell decomposition from the flag variety. Consequently, the map on cohomology

$$
H^{\star}(G / B) \rightarrow H^{\star}\left(X_{w}\right)
$$

induced by including $X_{w} \hookrightarrow G / B$ is surjective with kernel

$$
I_{w}:=\mathbb{k} \text {-span of }\left\{\sigma_{u}: u \not \leq w\right\} .
$$

This gives rise to the presentation

$$
\begin{aligned}
H^{\star}\left(X_{w}\right) & \cong H^{\star}(G / B) / I_{w} & & \text { working over } \mathbb{Z} \\
& \cong \mathbb{k}[V] /\left(I_{w}+\mathbb{k}[V]_{+}^{W}\right) & & \text { working over } \mathbb{k} .
\end{aligned}
$$

This presentation (1.3) involves a generating set (1.2) for $I_{w}$ with at most $|W|$ generators. However, this generating set for $I_{w}$ is wasteful in that it not only generates $I_{w}$ as an ideal but actually spans it $\mathbb{k}$-linearly within $H^{\star}(G / B)$.

Therefore, a basic question is to request more efficient generating sets for $I_{w}$. For type $A_{n-1}$, earlier work GR02 reduced the $|W|=n$ ! upper bound on the number of generators for $I_{w}$ to a polynomial bound of $n^{2}$ for the class of Schubert varieties $X_{w}$ defined by inclusions; this class includes all smooth $X_{w}$. Moreover, for a certain subclass of smooth Schubert varieties $X_{w}$ considered originally by Ding [D97, D01, they gave a smaller generating set for these $I_{w}$ having only $n$ generators. This latter result was applied in DMR07 to classify these varieties up to isomorphism and homeomorphism (the generating set (1.2) having proved too unwieldy). One motivation for this work arose from the desire to extend this classification to general 
Schubert varieties of type $A$. Experience suggests that presentations for $I_{w}$ that are as simple as possible are best for this purpose.

The question of finding simple presentations of $I_{w}$ for other root systems appears to have been less studied.

The main goal of this paper is to give a concise and root-system uniform extension of Borel's presentation that produces for arbitrary $w \in W$ an abbreviated list of generators for $I_{w}$. Our first main result, Theorem 1.1, achieves this via a strong restriction on the descent set

$$
\operatorname{Des}(u):=\{s \in S: \ell(u s)<\ell(u)\}
$$

of the elements $u$ in $W$ that index elements in our list of ideal generators $\sigma_{u}$ for $I_{w}$. We will need the following definitions, the first two of which are standard:

- An element $u \in W$ is grassmannian if $|\operatorname{Des}(u)| \leq 1$.

- An element $v \in W$ is bigrassmannian if both $v$ and $v^{-1}$ are grassmannian.

- Given $w \in W$, the essential set for $w$, denoted $\mathcal{E}(w)$, is the set of $u \in W$ which are minimal in the Bruhat order among those not below $w$.

The nomenclature "essential set" for $\mathcal{E}(w)$ is justified in Proposition 4.6, where we give a new characterization of Fulton's essential set [F92 for the case of the symmetric group $W=S_{n}$. Indeed, $\mathcal{E}(w)$ has been previously studied from a different point of view: a result of Lascoux and Schützenberger [LS96, Théorème 3.6], implicit in our first main result below, shows that the elements in $\mathcal{E}(w)$ are bigrassmannian for any $w$ in $W \underline{1}$

Theorem 1.1. For any $w \in W$, working in a field $\mathbb{k}$ of characteristic zero, the ideal $I_{w}$ defining $H^{\star}\left(X_{w}\right)$ as a quotient of $H^{\star}(G / B)$ is generated by the cohomology classes $\sigma_{u}$ where $u \leq \mathbb{w}$ and $u$ is grassmannian.

More precisely, $I_{w}$ is generated by the classes $\sigma_{u}$ indexed by those grassmannian $u$ for which there exist some bigrassmannian $v$ in $\mathcal{E}(w)$ satisfying both $u \geq v$ and $\operatorname{Des}(u)=\operatorname{Des}(v)$.

In type $A$, a similar result was obtained by Akyildiz, Lascoux, and Pragacz ALP92, Theorem 2.2]. Specifically, they prove the first sentence of Theorem 1.1. though they do not address the strengthening given by the second sentence. Their methods are mainly geometric, as opposed to our essentially combinatorial arguments. Their work provides, to our knowledge, the first inroads towards an abbreviated generating set for $I_{w}$.

Theorem 1.1 replaces the general upper bound of $|W|$ on the number of generators needed for $I_{w}$ with the bound

$$
\sum_{s \in S}\left[W: W_{S \backslash\{s\}}\right]
$$

where, for any subset $J \subset S, W_{J}$ denotes the parabolic subgroup of $W$ generated by $J$. Our theorem is deduced in Section 2 from a more general result (Theorem 2.4) that applies to Hiller's extension [H82, Chapter IV] of Schubert calculus as introduced by Bernstein, Gelfand, and Gelfand BGG73 and Demazure D73 to the coinvariant algebras of finite reflection groups $W$.

\footnotetext{
${ }^{1}$ For Lascoux and Schützenberger this arises in their work (see also Geck and Kim GK97 and Reading [R02]) on seeking efficient encodings of the strong Bruhat order; it is perhaps not surprising that it should arise in our search for efficient cohomology presentations as well.
} 
Section 2 explains and proves Theorem 1.1. In Section 3, we exploit the particular form of our generators to derive a straightforward extension to Schubert varieties in any partial flag manifold $G / P$ associated to a parabolic subgroup $P$ of $G$.

Section 4 examines more closely Theorem 1.1 in type $A_{n-1}$. Here one can take $G=G L_{n}(\mathbb{C})$, with $B$ the subgroup of invertible upper triangular matrices, $T$ the invertible diagonal matrices, and $W=S_{n}$ the permutation matrices. The bound on generators for $I_{w}$ in (1.4) becomes $2^{n}$, at least a practical improvement on $|W|=n$ !. More importantly, one can be even more explicit and efficient in the generating sets for $I_{w}$.

Identify points of $G / B$ with complete flags

$$
\langle 0\rangle \subset V_{1} \subset \cdots \subset V_{n-1} \subset \mathbb{C}^{n} .
$$

Under this identification, each Schubert variety $X_{w}$ is the set of flags satisfying certain specific conditions derived from $w$ of the form $\operatorname{dim}_{\mathbb{C}}\left(V_{r} \cap \mathbb{C}^{s}\right) \geq t$. The bigrassmannians $v$ comprising $\mathcal{E}(w)$ correspond to Fulton's essential Schubert conditions, a minimal list of such conditions defining the Schubert variety $X_{w}$. Our second main result (Theorem 4.8) provides for each bigrassmannian $v$ in $W=S_{n}$ a generating set for the ideal

$$
J_{v}:=\mathbb{k}-\operatorname{span} \text { of }\left\{\sigma_{u}: u \geq v\right\}
$$

in type $A$ that is smaller than the one used as a general step (Theorem 2.3) in the proof of Theorem 1.1 for arbitrary finite Coxeter groups $W$. Our proof of Theorem 4.8 is based on symmetric function identities that we devise for this purpose.

Therefore, concatenating these generating sets for $J_{v}$ gives a generating set for

$$
I_{w}=\sum_{v \in \mathcal{E}(w)} J_{v}
$$

that is smaller than the one provided by Theorem 1.1. We remark that this result subsumes (and slightly improves upon; see Example 4.9) the generating set of size $n^{2}$ given by GR02] in the case of Schubert varieties defined by inclusions.

Actually, we conjecture that this smaller generating set for $J_{v}$ in type $A$ is minimal (although the generating set (1.6) for $I_{w}$ obtained by concatenation is not always minimal; see Example 4.12). The significance of this minimality conjecture, as explained in Section 4.5. is that it implies an exponential lower bound of at least

$$
\left(\begin{array}{l}
n / 2 \\
n / 4
\end{array}\right) \sim \frac{\sqrt{2}^{n+2}}{\sqrt{\pi n}}
$$

on the number of generators needed for $I_{w}$, accompanying our exponential upper bound of $2^{n}$. Thus one would not be able to expect short presentations for $H^{*}\left(X_{w}\right)$ in general, at least in type $A$.

\section{Proof of Theorem 1.1}

As in Section 1 , let $\mathbb{k}$ be a field of characteristic zero. For $v, w$ in $W$, define two $\mathbb{k}$-linear subspaces $J_{v}, I_{w}$ of the cohomology $H^{\star}(G / B)$ with $\mathbb{k}$ coefficients:

$$
\begin{aligned}
& J_{v}:=\mathbb{k} \text {-span of }\left\{\sigma_{u}: u \geq v\right\}, \\
& I_{w}:=\mathbb{k}-\operatorname{span} \text { of }\left\{\sigma_{u}: u \not \leq w\right\}\left(=\sum_{v \in \mathcal{E}(w)} J_{v}\right) .
\end{aligned}
$$


Recall that the essential set $\mathcal{E}(w)$ is the set of all Bruhat-minimal elements of $\{v \in W: v \not \leq w\}$.

The Schubert cell decomposition of $G / B$ shows that both of these $\mathbb{k}$-subspaces are actually ideals in the cohomology ring $H^{\star}(G / B)$ : one has that $I_{w}$ (respectively $\left.J_{v}\right)$ is the kernel of the surjection $H^{\star}(G / B) \rightarrow H^{\star}(X)$ induced by the inclusion of the $B$-stable subvariety $X \subseteq G / B$, where $X=X_{w}$ (respectively $X=\bigcup_{u \nsucceq v} X_{u}$ ).

Also recall from Section 1 the following important property of bigrassmannians in the Bruhat order on Coxeter groups, originally due to Lascoux and Schützenberger [LS96, Théorème 3.6] and Geck and Kim GK97, Lemma 2.3 and Theorem 2.5].

Lemma 2.1. For any Coxeter system $(W, S)$ and $w$ in $W$, every element of $\mathcal{E}(w)$ is bigrassmannian.

See Section 4.2 below for a further interpretation of $\mathcal{E}(w)$ when $W$ is a Weyl group of type $A_{n-1}$. As a consequence of Lemma 2.1 and (2.1), finding generators of $J_{v}$ for bigrassmannian $v$ automatically gives generators for the ideals $I_{w}$.

We will actually work at the level of generality of irreducible Coxeter systems $(W, S)$ with $W$ finite, using Hiller's version $\mathrm{H} 82$ of the Schubert calculus BGG73, D73 for coinvariant algebras. This emphasizes that the arguments of this section and the next only depend on Coxeter combinatorics and formal properties of divided difference operators and coinvariant algebras. Here we review the relevant facts from [H82, Chapter IV].

Let $W$ be a finite and irreducible Coxeter group and $V$ its reflection representation. One then picks a (possibly non-crystallographic) root system $\Phi$ for $W$ as follows: $\Phi \subset V^{\star}$ is any $W$-stable choic 2 of a set of a linear functionals $\alpha$ and $-\alpha$ such that the perpendicular spaces $\alpha^{\perp}$ in $V$ run through the reflecting hyperplanes of $W$. These reflecting hyperplanes divide $V$ into chambers; we pick one which we call the dominant chamber $\mathcal{C}$. One declares the positive roots $\Phi^{+} \subseteq \Phi$ to be the roots which are positive on this chamber. The Coxeter generators $S$ of $W$ are declared to be the reflections across the hyperplanes which are facets of $\mathcal{C}$. Among the positive roots are the simple roots $\Pi=\left\{\alpha_{s}\right\}_{s \in S}$, where $\alpha_{s}$ is the positive root vanishing on the reflecting hyperplane of $s$.

With these choices, one defines for each $w \in W$ the Hiller Schubert polyno$\operatorname{mia} \sqrt[3]{3} S_{w}$ in $\mathbb{k}[V]=\operatorname{Sym}\left(V^{\star}\right)$ by

$$
S_{w}:=\partial_{w^{-1} w_{0}} S_{w_{0}},
$$

where $w_{0}$ is the unique longest element in $W$, for which one declares that

$$
S_{w_{0}}:=\frac{1}{|W|} \prod_{\alpha \in \Phi^{+}} \alpha .
$$

The images of the polynomials $S_{w}$ as $w$ ranges over all of $W$ will form a basis for the coinvariant algebra $\mathbb{k}[V] /\left(\mathbb{k}[V]_{+}^{W}\right)$. Here the divided difference operators $\partial_{u}$ on $\mathbb{k}[V]$ are defined first for $s \in S$ by

$$
\partial_{s}(f):=\frac{f-s(f)}{\alpha_{s}}
$$

\footnotetext{
${ }^{2}$ Note that this may require coefficients in a subfield $\mathbb{k}$ of $\mathbb{R}$ strictly larger than $\mathbb{Q}$ when $W$ is not crystallographic.

3 "BGG/Demazure Schubert polynomial" would be as fair, but for us the main point is to distinguish these polynomials from the type $A$ Lascoux-Schützenberger version forthcoming in Section 4 , which are not the same as elements of $k[V]$, although equivalent $\bmod k[V]_{+}^{W}$.
} 
and then for any $u \in W$ of Coxeter length $\ell=\ell(u)$ by

$$
\partial_{u}:=\partial_{s_{i_{1}}} \cdots \partial_{s_{i_{\ell}}}
$$

where $u=s_{i_{1}} \cdots s_{i_{\ell}}$ is any choice of a (reduced) decomposition expressing $u$ in terms of the generators $s \in S$.

The relation to a generalized flag manifold $G / B$ is that $G, B$, and $T$ come equipped with a (crystallographic) root system and Weyl group $W$. Let $\mathfrak{h} \subset \mathfrak{b} \subset \mathfrak{g}$ be the Lie algebras associated to $T \subset B \subset G$. Take the reflection representation $V$ to be $V=\mathfrak{h}$ (or more generally $V=\mathbb{k} \otimes_{\mathbb{Z}} X(T)$ ) and the root system $\Phi$ to be the set of weights of the adjoint representation of $\mathfrak{g}$ acting on itself, with $\Phi^{+}$the weights of the action of $\mathfrak{g}$ on $\mathfrak{b}$. In this case, it was proven in BGG73, D73 that the element $S_{w} \in \mathbb{k}[\mathfrak{h}]$ is a lift under the surjection

$$
\mathbb{k}[\mathfrak{h}] \rightarrow \mathbb{k}[\mathfrak{h}] /\left(\mathbb{k}[\mathfrak{h}]_{+}^{W}\right) \quad\left(\cong H^{\star}(G / B)\right)
$$

of the Schubert cohomology class $\sigma_{w}$ in $H^{\ell(w)}(G / B)$, which is Kronecker dual to the fundamental homology class $\left[X_{w}\right]$ of $X_{w}$ in $H_{\ell(w)}(G / B)$ and Poincaré dual to the fundamental homology class $\left[X_{w_{0} w}\right]$ in $H_{\ell\left(w_{0}\right)-\ell(w)}(G / B)$.

We collect basic properties of the divided difference operators $\partial_{s}$ and Schubert polynomials $S_{w}$ that we need below.

(a) Leibniz rule: $\partial_{s}(f g)=\partial_{s}(f) \cdot g+s(f) \partial_{s}(g)$.

(b) $\partial_{s}(f)=0$ if and only if $s(f)=f$. In the case where $f=S_{w}$ for some $w$, then $\partial_{s}\left(S_{w}\right)=0$ if and only if $\ell(w s)>\ell(w)$, or equivalently if and only if $s \notin \operatorname{Des}(w)$.

(c) Consequently, $\partial_{s}$ preserves the ideal $I=\mathbb{k}[V]_{+}^{W}$ generated by the $W$ invariants of positive degree in $\mathbb{k}[V]$.

(d)

$$
\partial_{u} \partial_{v}= \begin{cases}\partial_{u v} & \text { if } \ell(u)+\ell(v)=\ell(u v), \\ 0 & \text { otherwise. }\end{cases}
$$

(e) If $\ell(w)=\ell\left(w^{\prime}\right)$, then $\partial_{w^{\prime}} S_{w}=\delta_{w, w^{\prime}}$. (By definition, $\delta_{a, b}=1$ if $a=b$ and $\delta_{a, b}=0$ otherwise.)

(f) Consequently, the Schubert structure constants $c_{u, v}^{w}$ which uniquely express

$$
S_{u} S_{v}=\sum_{\substack{w \in W: \\ \ell(w)=\ell(u)+\ell(v)}} c_{u, v}^{w} S_{w} \quad \bmod k[V]_{+}^{W}
$$

can be computed by the formula $c_{u, v}^{w}=\partial_{w}\left(S_{u} S_{v}\right)$.

(g) These structure constants also satisfy this sparsity rule:

$$
c_{u, v}^{w}=0 \text { unless } w \geq u, v \text { in Bruhat order. }
$$

This is a consequence of the Pieri formula [H82, §IV.3] for multiplying the $S_{u}$ by any of the degree one elements that generate $\mathbb{k}[V] /\left(\mathbb{k}[V]_{+}^{W}\right)$.

The key to the proof of Theorem 1.1 turns out to be the following lemma (perhaps of independent interest) about some further sparsity of the Schubert structure constants $c_{u, v}^{w}$ appearing in property $(f)$ above. Given $J \subset S$, recall that every $w$ in $W$ has a unique length-additive parabolic factorization

$$
w=u \cdot x
$$


where $x$ lies in the parabolic subgroup $W_{J}$ generated by $J$ and $u$ lies in the set $W^{J}$ of minimum-length coset representatives for $W / W_{J}$, characterized by the property that $\operatorname{Des}(u) \cap J=\varnothing$.

Lemma 2.2. Let $J \subset S$. Suppose $w, w^{\prime}$ in $W$ lie in the same coset $w W_{J}=w^{\prime} W_{J}$ so that $w=u \cdot x$ and $w^{\prime}=u \cdot x^{\prime}$ for some $u$ in $W^{J}$ and $x, x^{\prime}$ in $W_{J}$. Then $c_{u, x}^{w^{\prime}}=\delta_{w^{\prime}, w}=\delta_{x^{\prime}, x}$.

Proof. Using property (f) of divided differences above, one can rephrase the lemma as saying that, for any $w^{\prime} \in W$ with $w^{\prime}=u \cdot x^{\prime}$ where $x^{\prime} \in W_{J}$ and $\ell\left(x^{\prime}\right)=\ell(x)$ (so that $\left.\ell\left(w^{\prime}\right)=\ell(w)\right)$, one has

$$
\partial_{w^{\prime}}\left(S_{u} S_{x}\right)=\delta_{x, x^{\prime}}
$$

We prove (2.2) by induction on $\ell\left(x^{\prime}\right)$. In the base case where $\ell\left(x^{\prime}\right)=0$, one has $x^{\prime}=x=1$ and $w^{\prime}=w=u$, so the assertion (2.2) follows from property (e) above.

In the inductive step, let $\ell\left(x^{\prime}\right)>0$. Thus, there exists $s \in J$ with $\ell\left(x^{\prime} s\right)<\ell\left(x^{\prime}\right)$. Consequently,

$$
\ell\left(w^{\prime}\right)=\ell(u)+\ell\left(x^{\prime}\right)=\ell(u)+\ell\left(x^{\prime} s\right)+\ell(s) .
$$

Hence by properties (d), (a) and (b), one has, respectively,

$$
\begin{aligned}
\partial_{w^{\prime}}\left(S_{u} S_{x}\right) & =\partial_{u} \partial_{x^{\prime} s} \partial_{s}\left(S_{u} S_{x}\right) \\
& =\partial_{u} \partial_{x^{\prime} s}\left(\partial_{s}\left(S_{u}\right) \cdot S_{x}+s\left(S_{u}\right) \partial_{s}\left(S_{x}\right)\right) \\
& =\partial_{u} \partial_{x^{\prime} s}\left(S_{u} \partial_{s}\left(S_{x}\right)\right) .
\end{aligned}
$$

Now consider two cases.

Case 1. $\ell(x s)>\ell(x)$. Then property (b) says that $\partial_{s}\left(S_{x}\right)=0$. Using this in the last line of (2.3), one concludes that

$$
\partial_{w^{\prime}}\left(S_{u} S_{x}\right)=0=\delta_{x, x^{\prime}}
$$

since $\ell\left(x^{\prime} s\right)<\ell\left(x^{\prime}\right)$ implies $x \neq x^{\prime}$.

Case 2. $\ell(x s)<\ell(x)$. Then $\partial_{s}\left(S_{x}\right)=S_{x s}$, and

$$
\begin{aligned}
\partial_{w^{\prime}}\left(S_{u} S_{x}\right) & =\partial_{u} \partial_{x^{\prime} s}\left(S_{u} \partial_{s}\left(S_{x}\right)\right) \\
& \left.=\partial_{u} \partial_{x^{\prime} s}\left(S_{u} S_{x s}\right)\right) \\
& =\delta_{x s, x^{\prime} s} \\
& =\delta_{x, x^{\prime}},
\end{aligned}
$$

where the second-to-last equality applied the inductive hypothesis to $x^{\prime} s$ and $x s$.

We now use this lemma to find a smaller generating set for the ideal $J_{v}$ as defined in (2.1) based on the descent set $\operatorname{Des}(v)$. Working more generally, for any finite Coxeter group $W$ and a choice of root data for the Hiller Schubert calculus, define the following two $\mathbb{k}$-subspaces within the coinvariant algebra $\mathbb{k}[V] /\left(\mathbb{k}[V]_{+}^{W}\right)$ :

$$
\begin{aligned}
J_{v} & :=\mathbb{k} \text {-span of }\left\{S_{u}: u \geq v\right\}, \\
I_{w} & :=\mathbb{k} \text {-span of }\left\{S_{u}: u \not \leq w\right\}=\sum_{v \in \mathcal{E}(w)} J_{v} .
\end{aligned}
$$

Note that in this context we can appeal to property $(\mathrm{g})$ to see that $J_{v}$ and $I_{w}$ are actually ideals within the coinvariant algebra $\mathbb{k}[V] /\left(\mathbb{k}[V]_{+}^{W}\right)$. 
Theorem 2.3. Let $v$ be an element of a finite Coxeter group $W$ and $J$ a subset of $S$. Assume $v$ lies in $W^{J}$ or, equivalently, $\operatorname{Des}(v) \cap J=\varnothing$.

Then $J_{v}$ is generated as an ideal within the coinvariant algebra $\mathbb{k}[V] /\left(\mathbb{k}[V]_{+}^{W}\right)$ by the set

$$
\left\{S_{u}: u \in W^{J}, u \geq v\right\} .
$$

Proof. For any $t \in W^{J}$, let $J_{t}^{\prime}$ denote the ideal

$$
\left\{S_{u}: u \in W^{J}, u \geq t\right\} .
$$

We need to show $J_{v}^{\prime}=J_{v}$. Certainly $J_{v}^{\prime} \subseteq J_{v}$ by definition, so it remains to show the reverse inclusion.

Proceed by induction on the colength $\ell\left(w_{0}\right)-\ell(v)$ of $v$. In the base case where $v$ has colength $0, v=w_{0}$. Therefore the assumption $v \in W^{J}$ implies $J=\varnothing$, so $W^{J}=W$, and there is nothing to prove.

In the inductive step, given $w \geq v$, one must show that $S_{w}$ lies in $J_{v}^{\prime}$. Factor $w=u \cdot x$ uniquely with $u \in W^{J}$ and $x \in W_{J}$. We will repeatedly use the fact (see [BB05, §2.5]) that the map

$$
\begin{aligned}
W & \stackrel{P^{J}}{\longrightarrow} W^{J}, \\
w & \longmapsto u
\end{aligned}
$$

is order-preserving for the Bruhat order. In particular, since it was assumed that $w \geq v$ above, one has $u \geq v$ here.

By Lemma 2.2. one has

$$
\begin{aligned}
S_{u} S_{x} & =S_{w}+\sum_{w^{\prime}} c_{u, x}^{w^{\prime}} S_{w^{\prime}}, \quad \text { so that } \\
S_{w} & =S_{u} S_{x}-\sum_{w^{\prime}} c_{u, x}^{w^{\prime}} S_{w^{\prime}} .
\end{aligned}
$$

Here each $w^{\prime}$ appearing in the sums satisfies $w^{\prime} \geq u$ by property $(\mathrm{g})$, and hence if one factors $w^{\prime}=u^{\prime} \cdot x^{\prime}$ with $u^{\prime} \in W^{J}$ and $x^{\prime} \in W_{J}$, then $w^{\prime} \geq u$ implies $u^{\prime} \geq u$. But then Lemma 2.2 also says that $c_{u, x}^{w^{\prime}}=0$ unless $u^{\prime}>u(\geq v)$. Hence, by induction, for any $w^{\prime}$ with $S_{w^{\prime}}$ appearing with non-zero coefficient in the right hand sum, $J_{u^{\prime}}=J_{u^{\prime}}^{\prime}$ holds for the corresponding $u^{\prime}$ in the factorization $w^{\prime}=u^{\prime} \cdot x^{\prime}$. By definition, $J_{u^{\prime}}^{\prime} \subset J_{v}^{\prime}$, and since $S_{u}$ also lies in $J_{v}^{\prime}$ as $u \in W^{J}$, one concludes that $S_{w}$ lies in $J_{v}^{\prime}$, as desired.

Theorem 1.1 is then a special case of the following result, which is immediate from Lemma 2.1 and Theorem 2.3

Theorem 2.4. Let $W$ be a Coxeter system $(W, S)$ with $W$ finite, and let $w$ be an element of $W$. Then the ideal $I_{w}$ of the coinvariant algebra $\mathbb{k}[V] /\left(\mathbb{k}[V]_{+}^{W}\right)$, defined as in (2.4), is generated by the Schubert polynomials

$$
\left\{S_{u}: u \not \leq w \text {, and } u \text { is grassmannian }\right\} \text {. }
$$

More precisely, $I_{w}$ is generated by the set $\left\{S_{u}\right\}$ for those $u$ for which there exist some (bigrassmannian) $v$ in $\mathcal{E}(w)$ satisfying both $u \geq v$ and $\operatorname{Des}(u)=\operatorname{Des}(v)$. 


\section{Reducing PResentations in $H^{\star}(G / P)$ TO ThOse IN $H^{\star}(G / B)$}

We explain in this section how Theorem 2.4leads to a shorter presentation more generally for the cohomology of Schubert varieties in any partial flag manifold.

Given $J \subset S$, one has the parabolic subgroup $W_{J}$ of $W$ generated by $J$. One also has a corresponding parabolic subgroup $P_{J}$ of $G$, generated by the Borel subgroup $B$ together with representatives within $G$ that lift the elements $J \subset W=N_{G}(T) / T$. The Borel picture identifies the cohomology ring of the (generalized) partial flag manifold $G / P_{J}$ as the subring of $W_{J}$-invariants inside the cohomology of $G / B$. In other words, the quotient map

$$
G / B \stackrel{\pi}{\rightarrow} G / P_{J}
$$

induces the inclusion

$$
H^{\star}\left(G / P_{J}\right) \cong H^{\star}(G / B)^{W_{J}} \stackrel{i}{\hookrightarrow} H^{\star}(G / B) .
$$

Recall that $W^{J}$ denotes the set of minimum-length coset representatives for $W / W_{J}$. Inside $H^{\star}(G / B)$, the cohomology classes $\left\{\sigma_{w}: w \in W^{J}\right\}$ lie in this $W_{J^{-}}$ invariant subalgebra $H^{\star}(G / B)^{W_{J}}$ and form a $\mathbb{k}$-basis identified with the $\mathbb{k}$-basis of Schubert cohomology classes $\sigma_{w W_{J}}$ for $H^{\star}\left(G / P_{J}\right)$. One also has that the pre-image of Schubert varieties in $G / P_{J}$ are certain Schubert varieties of $G / B$ : specifically,

$$
\pi^{-1}\left(X_{w W_{J}}\right)=X_{w_{\max }}
$$

where $w_{\max }$ is the unique maximum-length coset representative in $w W_{J}$.

Working more generally with the Hiller Schubert calculus for any finite Coxeter system $(W, S)$, the inclusion (3.1) generalizes to the inclusion

$$
\mathbb{k}[V]^{W_{J}} / \mathbb{k}[V]_{+}^{W} \mathbb{k}[V]^{W_{J}} \cong\left(\mathbb{k}[V] /\left(\mathbb{k}[V]_{+}^{W}\right)\right)^{W_{J}} \stackrel{i}{\hookrightarrow} \mathbb{k}[V] /\left(\mathbb{k}[V]_{+}^{W}\right) .
$$

The first isomorphism shown in (3.2) is a consequence of the fact that one has an averaging map

$$
\begin{aligned}
\mathbb{k}[V] & \stackrel{\rho}{\longrightarrow} \mathbb{k}[V]^{W_{J}}, \\
f & \longmapsto \frac{1}{\left|W_{J}\right|} \sum_{w \in W_{J}} w(f),
\end{aligned}
$$

which provides a $\mathbb{k}[V]^{W_{J}}$-linear (and hence also $\mathbb{k}[V]^{W}$-linear) retraction map for $i$,

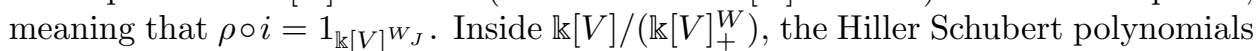
$\left\{S_{w}: w \in W^{J}\right\}$ lie in this $W_{J}$-invariant subalgebra $\left(\mathbb{k}[V] /\left(\mathbb{k}[V]_{+}^{W}\right)\right)^{W_{J}}$ and provide a $\mathbb{k}$-basis for it.

The retraction in (3.3) also provides the relation between the cohomology presentations for the Schubert varieties $X_{w W_{J}}$ and $X_{w_{\max }}$. Recall that when one has an inclusion of rings $R \stackrel{i}{\hookrightarrow} \hat{R}$, one can relate ideals of $R$ and $\hat{R}$ by the operations of extension and contraction: given an ideal $I$ in $R$, its extension $\hat{R} I$ to $\hat{R}$ is the ideal it generates in $\hat{R}$, and given an ideal $\hat{I}$ of $\hat{R}$, its contraction to $R$ is the intersection $\hat{I} \cap R$. Say that the inclusion $R \stackrel{i}{\hookrightarrow} \hat{R}$ is a split inclusion if it has an $R$-linear retraction $\hat{R} \stackrel{\rho}{\rightarrow} R$, meaning that $\rho \circ i=1_{R}$. The following proposition about this situation is straightforward and well known.

Proposition 3.1. Assume $R \stackrel{i}{\hookrightarrow} \hat{R}$ is a split inclusion and $\hat{I}$ is an ideal of $\hat{R}$ which is generated by its contraction $I:=\hat{I} \cap R$ to $R$.

Then a set of elements $\left\{g_{\alpha}\right\}$ lying in $R$ generate $\hat{I}$ as an ideal of $\hat{R}$ if and only if the same elements $\left\{g_{\alpha}\right\}$ generate $I=\hat{I} \cap R$ as an ideal of $R$. 
We will apply this proposition to the split inclusion (3.2) and to the ideals

$$
\begin{aligned}
& I=I_{w W_{J}}:=\mathbb{k} \text {-span of }\left\{S_{u}: u \in W^{J}, u W_{J} \not \leq w W_{J}\right\} \subset\left(\mathbb{k}[V] /\left(\mathbb{k}[V]_{+}^{W}\right)\right)^{W_{J}}=R, \\
& \hat{I}=I_{w_{\max }}:=\mathbb{k} \text {-span of }\left\{S_{u}: u \in W, u \not \leq w_{\max }\right\} \subset \mathbb{k}[V] /\left(\mathbb{k}[V]_{+}^{W}\right)=\hat{R},
\end{aligned}
$$

which in the case where $(W, S)$ comes from an algebraic group $G$ have the following interpretations as kernels:

$$
\begin{aligned}
& I_{w W_{J}}=\operatorname{ker}\left(H^{\star}\left(G / P_{J}\right) \stackrel{i^{\star}}{\rightarrow} H^{\star}\left(X_{w W_{J}}\right)\right), \\
& I_{w_{\max }}=\operatorname{ker}\left(H^{\star}(G / B) \stackrel{i^{\star}}{\rightarrow} H^{\star}\left(X_{w_{\max }}\right)\right) .
\end{aligned}
$$

Borel's picture already gives a very short presentation for $H^{\star}\left(G / P_{J}\right)$, or more generally $\left(\mathbb{k}[V] /\left(\mathbb{k}[V]_{+}^{W}\right)\right)^{W_{J}}$, as we now explain. The isomorphism in (3.2) says that a presentation of $\left(\mathbb{k}[V] /\left(\mathbb{k}[V]_{+}^{W}\right)\right)^{W_{J}}$ is equivalent to a presentation of the quotient $\mathbb{k}[V]^{W_{J}} / \mathbb{k}[V]_{+}^{W} \mathbb{k}[V]^{W_{J}}$. Since both $W_{J}$ and $W$ are finite reflection groups acting on $V$, their invariant rings are both polynomial algebras

$$
\begin{aligned}
\mathbb{k}[V]^{W_{J}} & =\mathbb{k}\left[g_{1}, \ldots, g_{n}\right], \\
\mathbb{k}[V]^{W} & =\mathbb{k}\left[f_{1}, \ldots, f_{n}\right],
\end{aligned}
$$

and hence the quotient can be presented as a graded complete intersection ring:

$$
\begin{aligned}
\left(\mathbb{k}[V] /\left(\mathbb{k}[V]_{+}^{W}\right)\right)^{W_{J}} & \cong \mathbb{k}[V]^{W_{J}} / \mathbb{k}[V]_{+}^{W_{+}} \mathbb{k}[V]^{W_{J}} \\
& \cong \mathbb{k}\left[g_{1}, \ldots, g_{n}\right] /\left(f_{1}, \ldots, f_{n}\right) .
\end{aligned}
$$

Thus we only need to provide generators for the ideal $I_{w W_{J}}$.

Theorem 3.2. Let $(W, S)$ be a finite Coxeter system, with $J \subseteq S$ and $w$ in $W$, and let $w_{\max }$ be the unique maximum-length representative of $w W_{J}$. Consider the inclusion

$$
R:=\left(\mathbb{k}[V] /\left(\mathbb{k}[V]_{+}^{W}\right)\right)^{W_{J}} \quad \stackrel{i}{\hookrightarrow} \quad \hat{R}:=\mathbb{k}[V] /\left(\mathbb{k}[V]_{+}^{W}\right) .
$$

Then the following hold:

(i) The essential set $\mathcal{E}\left(w_{\max }\right)$ lies entirely in $W^{J}$.

(ii) The ideal $I_{w_{\max }}$ of $\hat{R}$ is generated by its contraction $I_{w_{\max }} \cap R$.

(iii) This contraction is the same as $I_{w W_{J}}$.

(iv) The set

$$
\bigcup_{v \in \mathcal{E}(w)}\left\{S_{u}: u \geq v, \operatorname{Des}(u)=\operatorname{Des}(v)\right\}
$$

both generates $I_{w_{\max }}$ as an ideal of $\hat{R}$ and also generates the contraction $I_{w W_{J}}$ as an ideal of $R$.

Proof. For assertion (i), assume for the sake of contradiction that $v \in \mathcal{E}\left(w_{\max }\right)$ and that $v s<v$ for some $s$ in $J$. Since $v$ is Bruhat-minimal among the elements not below $w_{\max }$, this implies $v s \leq w_{\max }$. However $w_{\max } s<w_{\max }$ by maximality of $w_{\max }$ within $w W_{J}$, so the lifting property [BB05, Prop. 2.2.7, Cor. 2.2.8] of Bruhat order implies that $v \leq w_{\max }$, a contradiction.

For assertion (ii), apply Theorem 2.4 to $w_{\max }$ to conclude that $I_{w}$ is generated by the set $\left\{S_{u}\right\}$ for those $u$ for which there exist $v \in \mathcal{E}\left(w_{\max }\right)$ satisfying both $u \geq v$ 
and $\operatorname{Des}(u) \subseteq \operatorname{Des}(v)$. By (i), this forces $u$ to lie in $W^{J}$, so that $S_{u}$ is $W^{J}$-invariant and therefore lies in $R$.

For assertion (iii), from the definition (3.4) and the fact that $R$ has a $\mathbb{k}$-basis given by $\left\{S_{u}: u \in W^{J}\right\}$, it suffices to show that for any $u$ in $W^{J}$, one has $u W_{J} \leq w W_{J}$ if and only if $u \leq w_{\max }$. By definition, $u W_{J} \leq w W_{J}$ if and only if $u \leq w_{\min }$, where $w_{\min }$ is the unique representative of $w W_{J}$ lying in $W^{J}$. The usual parabolic factorization $W=W^{J} \cdot W_{J}$ allows one to write a reduced word $\omega$ for $w_{\max }$ in the concatenated form

$$
\omega=\omega_{1} \cdot \omega_{2},
$$

where the prefix $\omega_{1}$ factors $w_{\min }$ and the suffix $\omega_{2}$ contains only generators in $J$. The subword characterization of Bruhat order BB05, Cor. 2.2.3] shows that $u \leq w_{\max }$ if and only if $u$ is factored by a reduced subword of this word $\omega$. This subword must necessarily use no generators from $J$ (since $u$ is in $W^{J}$ ) and hence must actually be a subword of $\omega_{1}$. Thus $u \leq w_{\max }$ if and only if $u \leq w_{\min }$, as desired.

Assertion (iv) then follows from assertions (ii), (iii) and Proposition 3.1 .

\section{Refinements in type $A$}

We further investigate the situation when $W$ is a Weyl group of type $A_{n-1}$, which exhibits extra features. One can:

- be more explicit about bigrassmannians and their essential sets $\mathcal{E}(w)$,

- produce even smaller generating sets for the ideals $I_{w}$ and $J_{v}$, which are conjecturally minimal in the case of $J_{v}$, and

- work with $\mathbb{Z}$ coefficients rather than over a field $\mathbb{k}$ of characteristic zero.

4.1. Schubert conditions and bigrassmannians. In type $A_{n-1}$, points in the variety $G / B$ are identified with complete flags of subspaces

$$
\langle 0\rangle \subset V_{1} \subset \cdots \subset V_{n-1} \subset \mathbb{C}^{n}
$$

having $\operatorname{dim}_{\mathbb{C}} V_{i}=i$. Pick as our particular base flag $\left\{\mathbb{C}^{i}\right\}_{i=0}^{n}$, where $\mathbb{C}^{i}$ is spanned by the first $i$ standard basis elements; this flag is fixed by the Borel subgroup $B$ consisting of the invertible upper-triangular matrices within $G:=G L_{n}(\mathbb{C})$. Picking the maximal torus $T$ of invertible diagonal matrices, one identifies the Weyl group $W=N_{G}(T) / T$ with the symmetric group $S_{n}$. The Coxeter generators $S$ for $W=$ $S_{n}$ associated to our Borel subgroup $B$ is the set of adjacent transpositions $S=$ $\{(1 \leftrightarrow 2),(2 \leftrightarrow 3), \ldots,(n-1 \leftrightarrow n)\}$.

The Schubert variety $X_{w}$ corresponding to a permutation $w=w_{1} w_{2} \cdots w_{n} \in S_{n}$ (written in one-line notation) can be defined as the subvariety of flags satisfying the conjunction of the Schubert conditions

$$
\operatorname{dim}_{\mathbb{C}}\left(V_{r} \cap \mathbb{C}^{s}\right) \geq t,
$$

where

$$
t=t_{r, s}(w):=\left|\left\{w_{1}, w_{2}, \ldots, w_{r}\right\} \cap\{1,2, \ldots, s\}\right|
$$

for all $r, s=1,2, \ldots, n-1$ is the rank function associated to $w$. Denote the condition (4.1) by $C_{r, s, t}$ (for arbitrary $t$, not necessarily of the form (4.2)). Note that $C_{r, s, t}$ is vacuous unless $t>r+s-n$.

The following explicit identification of bigrassmannian permutations is well known and straightforward. 
Lemma 4.1. The bigrassmannian permutations (other than the identity) are parameterized by $r, s, t$ with $1 \leq t \leq r, s \leq n$ and $t>r+s-n$. Let $v_{r, s, t, n}$ denote the unique bigrassmannian permutation $v_{1} \cdots v_{n} \in S_{n}$ such that

- $\operatorname{Des}(v)=\{(r \leftrightarrow r+1)\}$,

- $\operatorname{Des}\left(v^{-1}\right)=\{(s \leftrightarrow s+1)\}$, and

- $v_{t}=s+1$

Then explicitly we have

$$
\begin{aligned}
& v_{r, s, t, n}:=(1,2, \ldots, t-1, \\
& s+1, s+2, \ldots, s+r-t+1, \\
& t, t+1, t+2, \ldots, s, \\
& s+r-t+2, s+r-t+3, \ldots, n) .
\end{aligned}
$$

There is a simple relation between these Schubert conditions $C_{r, s, t}$ and the bigrassmannian permutations in $W=S_{n}$ :

Proposition 4.2. Let $w \in S_{n}$. Then the Schubert condition $C_{r, s, t}$ is satisfied by all flags in $X_{w}$ if and only if $v_{r, s, t, n} \not \leq w$.

Proof. Note that $C_{r, s, t}$ is a Schubert condition on $X_{w}$ if and only if $t_{r, s}(w) \geq t$. It is then straightforward to check that the latter is equivalent to $v_{r, s, t, n} \not \leq w$ by using the tableau criterion [BB05, Theorem 2.6.3] for comparing elements in the Bruhat ordering.

Note that imposing an arbitrary conjunction of Schubert conditions on complete flags cuts out a $B$-stable subvariety of $G / B$, but this subvariety may be a reducible union of Schubert varieties rather than a single Schubert variety $X_{w}$. However, in type $A$, when one imposes a single Schubert condition, the result is always a (single) Schubert variety. This fact can be traced to special properties of the Bruhat order in type $A$, first identified by Lascoux and Schützenberger [LS96, and exploited further by Geck and Kim [GK97] and Reading [R02]. To explain this, we first recall some terminology.

Definition 4.3. In a poset $P$, say that an element $v$ is a dissector of $P$ if there exists a (necessarily unique) element $w$ in $P$ for which $P$ decomposes as the disjoint union of the principal order filter above $v$ and the principal order ideal below $w$ :

$$
P=\{u \in P: u \geq v\} \quad \sqcup \quad\{u \in P: u \leq w\} .
$$

Say that an element $a$ in a poset $P$ (which need not be a lattice) is join-irre-ducible if there does not exist a subset $X \subset P$ with $a \notin X$ such that $a$ is the least element among all upper bounds for $X$ in $P$.

There are two subtle issues to point out in this definition of join-irreducibles. First, when the finite poset is a lattice, an element is join-irreducible if and only if it covers a unique element. However, for non-lattices, one can have join-irreducibles that cover more than one element. For example, the strong Bruhat order in type $A_{2}$ has four non-minimal, non-maximal elements, each of which is join-irreducible, but two of them cover two elements. Second, all of the posets that we will consider have a unique least element (e.g. in Bruhat order on $W$, the least element is the identity of $W$ ), and this least element is not considered join-irreducible because it is the least element among all upper bounds for the empty set $X=\varnothing$. 
Theorem 4.4 ([LS96, GK97, R02]). $\quad$ (i) In any finite poset, every dissector is join-irreducible. When the poset is the Bruhat order for a Coxeter system $(W, S)$ of type $A, B, H_{3}, H_{4}$ or $I_{2}(m)$, the converse holds: the joinirreducible elements are exactly the dissectors.

(ii) In the Bruhat order for any finite Coxeter system $(W, S)$, every join-irreducible element is bigrassmannian. In type A, the converse holds: the (nonidentity) bigrassmannian elements are exactly the join-irreducibles.

In particular, in type $A$, for every bigrassmannian $v$ in $W$ there exists a (necessarily unique) element $w$ in $W$ for which $\mathcal{E}(w)=\{v\}$.

Corollary 4.5. In type $A_{n-1}$, a single Schubert condition $C_{r, s, t}$ on the flags in $G / B$ cuts out the Schubert variety $X_{w_{r, s, t, n}}$ in $G / B$, where $w_{r, s, t, n}$ is the unique element with $\mathcal{E}\left(w_{r, s, t, n}\right)=\left\{v_{r, s, t, n}\right\}$ as in Theorem 4.4.

Thus in type $A_{n-1}$, for any bigrassmannian $v_{r, s, t, n}$ one has equality of the two ideals $J_{v_{r, s, t, n}}=I_{w_{r, s, t, n}}$ within the coinvariant algebra $\mathbb{k}[V] /\left(\mathbb{k}[V]_{+}^{W}\right)$.

We remark that, as with $v_{r, s, t, n}$, one knows $w_{r, s, t, n}$ explicitly (see [R02, §8]):

$$
\begin{gathered}
w_{r, s, t, n}=(n, n-1, \ldots,(n-r+t+1), \\
s, s-1, \ldots, s-t+1, \\
n-r+t, n-r+t-3, \ldots, s+1, \\
s-t, s-t-1, \ldots, 1) .
\end{gathered}
$$

4.2. Bigrassmannians and essential Schubert conditions. Next, we explain the relation between what we have called the essential set $\mathcal{E}(w)$ for $w$ and Fulton's essential set of Schubert conditions for $X_{w}$.

Note that there are implications among the various Schubert conditions $C_{r, s, t}$. Fulton introduced the essential set of a permutation, a set of coordinates $\left\{\left(r_{i}, s_{i}\right)\right\}$ $\subset n \times n$ which give an inclusion-minimal subset of Schubert conditions $C_{r_{i}, s_{i}, t}$ with $t=t_{r_{i}, s_{i}}(w)$ that suffice to define $X_{w}$ as a subset of the flag manifold. (See further remarks in Example4.7below.) Correspondingly, we call these Schubert conditions the essential Schubert conditions for $X_{w}$; see [F92, §3], [FP98, pp. 20-21], and EL96, §2].

Proposition 4.6. The Schubert condition $C_{r, s, t}$ implies the Schubert condition $C_{r^{\prime}, s^{\prime}, t^{\prime}}$ if and only if $v_{r, s, t, n} \leq v_{r^{\prime}, s^{\prime}, t^{\prime}, n}$ in Bruhat order.

Therefore in type $A_{n-1}$, Fulton's essential set of Schubert conditions $C_{r, s, t}$ for $X_{w}$ correspond bijectively to the elements of the essential set $\mathcal{E}(w)$ for $w$ defined for a general Coxeter group.

Proof. For the first assertion, note that the Schubert cell decomposition for $X_{w}$ and Theorem 4.4 give the following:

$$
\begin{array}{rlrl}
C_{r, s, t} \text { implies } C_{r^{\prime}, s^{\prime}, t^{\prime}} & \Leftrightarrow & X_{w_{r, s, t, n}} \subseteq X_{w_{r^{\prime}, s^{\prime}, t^{\prime}, n}} \\
& \Leftrightarrow \quad\left\{u \in W: u \leq w_{r, s, t, n}\right\} \subseteq\left\{u \in W: u \leq w_{r^{\prime}, s^{\prime}, t^{\prime}, n}\right\} \\
& \Leftrightarrow \quad\left\{u \in W: u \geq v_{r, s, t, n}\right\} \supseteq\left\{u \in W: u \geq v_{r^{\prime}, s^{\prime}, t^{\prime}, n}\right\} \\
& \Leftrightarrow \quad v_{r, s, t, n} \leq v_{r^{\prime}, s^{\prime}, t^{\prime}, n} .
\end{array}
$$

The second assertion follows immediately from the first.

Example 4.7. In order to be explicit about the bijection asserted in Proposition 4.6. it will be convenient for us to use a slight adaptation of Fulton's essential 
set. This bijection can be inferred from the discussion in [FP98] and GR02] (our conventions are in line with those in the latter text), and one can also thereby give an explicit bijection between our essential set and Fulton's essential set as originally defined.

Given $w=w_{1} w_{2} \cdots w_{n} \in S_{n}$, draw an $n \times n$ matrix of "bubbles" $\circ$. Replace the bubbles in positions $\left(i, w_{i}\right)$ with an $\times$ and erase all bubbles in the "hooks" weakly below and (non-standardly) to the left of each $\times$. The diagram of $w$, denoted $\mathcal{D}(w)$, consists of all bubbles left, which are those not in any hook. Under this convention $|\mathcal{D}(w)|=\ell\left(w_{0} w\right)$. This reflects the fact that our diagram is the left $\leftrightarrow$ right mirror image of the standard diagram of $w_{0} w$; see [FP98, p. 11]. Fulton's essential set is then defined as the subset of $\mathcal{D}(w)$ such that neither $(i+1, j)$ nor $(i, j-1)$ is in $\mathcal{D}(w)$. Let us denote Fulton's essential set by $\mathcal{E}_{\text {Fulton }}(w)$. The desired bijection between bubbles in $\mathcal{E}_{\text {Fulton }}(w)$ and $\mathcal{E}(w)$ sends the essential bubble with (row, column) indices $(r, s+1)$ to the bigrassmannian $v_{r, s, t, n}$, where $t$ is the number of bubbles weakly above the essential one in the same column.

For example, let $w=425163$. The figure below shows the positions $\left(i, w_{i}\right)$ marked with an $\times$, as well as the bubbles in the diagram $D(w)$ shown as $\bullet$ or $\circ$ depending upon whether or not they lie in $\mathcal{E}_{\text {Fulton }}(w)$ :

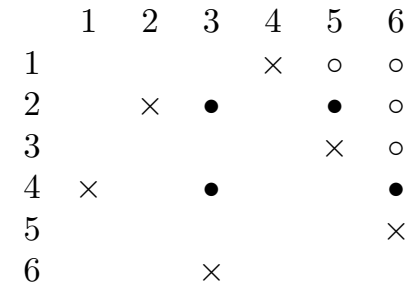

The following table then summarizes the bijection between the bubbles lying in Fulton's essential set $\mathcal{E}_{\text {Fulton }}(w)$, the essential Schubert conditions defining $X_{w}$, and the bigrassmannians that comprise $\mathcal{E}(w)$.

\begin{tabular}{|c|c|c|}
\hline $\begin{array}{c}(r, s+1)=(\text { row }, \text { column }) \\
\text { for bubble in } \mathcal{E}_{\text {Fulton }}(w)\end{array}$ & $\begin{array}{c}\text { Schubert condition } C_{r, s, t}: \\
\operatorname{dim} V_{r} \cap \mathbb{C}^{s} \geq t\end{array}$ & $\begin{array}{c}\text { bigrassmannian } v=v_{r, s, t, n} \\
\text { in } \mathcal{E}(w)\end{array}$ \\
\hline \hline$(2,3)$ & $\operatorname{dim} V_{2} \cap \mathbb{C}^{2} \geq 1$ & 341256 \\
\hline$(2,5)$ & $\begin{array}{c}\operatorname{dim} V_{2} \cap \mathbb{C}^{4} \geq 2 \\
\left(\text { i.e. } V_{2} \subset \mathbb{C}^{4}\right)\end{array}$ & 152346 \\
\hline$(4,3)$ & $\begin{array}{c}\operatorname{dim} V_{4} \cap \mathbb{C}^{2} \geq 2 \\
\left(\text { i.e. } \mathbb{C}^{2} \subset V_{4}\right)\end{array}$ & 134526 \\
\hline \multirow{2}{*}{$(4,6)$} & $\begin{array}{c}\operatorname{dim} V_{4} \cap \mathbb{C}^{5} \geq 4 \\
\left(\text { i.e. } V_{4} \subset \mathbb{C}^{5}\right)\end{array}$ & 123645 \\
\hline
\end{tabular}

4.3. Grassmannians and symmetric functions. In looking for generators for the ideals $J_{v_{r, s, t, n}}$ we wish to take advantage of symmetric function identities, so we briefly review the relation between symmetric functions and the Schubert calculus in type $A$. We also point out how the calculations may be performed over $\mathbb{Z}$ rather than a coefficient field $\mathbb{k}$ of characteristic zero. 
Let $J:=S \backslash\left\{s_{r}\right\}$ where $s_{r}=(r \leftrightarrow r+1)$, so that $W_{J}=S_{r} \times S_{n-r}$, and $G / P_{J}$ is the Grassmannian of $r$-planes in $\mathbb{C}^{n}$. The cohomology inclusion (3.1) or (3.2) remains valid working with coefficients in $\mathbb{Z}$ and becomes

$$
\begin{array}{lll}
H^{\star}\left(G / P_{J}\right) & \cong H(G / B)^{W_{J}} & \hookrightarrow H(G / B), \\
\mathbb{Z}[\mathbf{x}]^{W_{J}} / \mathbb{Z}[\mathbf{x}]_{+}^{W} \mathbb{Z}[\mathbf{x}]^{W_{J}} & \cong\left(\mathbb{Z}[\mathbf{x}] /\left(\mathbb{Z}[\mathbf{x}]_{+}^{W}\right)\right)^{W_{J}} & \hookrightarrow \mathbb{Z}[\mathbf{x}] /\left(\mathbb{Z}[\mathbf{x}]_{+}^{W}\right) .
\end{array}
$$

Here $\mathbb{Z}[\mathbf{x}]:=\mathbb{Z}\left[x_{1}, \ldots, x_{n}\right]$ is viewed as the symmetric algebra $\mathbb{Z}[V]$, where $V$ is no longer the irreducible reflection representation of dimension $n-1$ for $W=S_{n}$ but rather the natural permutation representation of dimension $n$. In order to work over $\mathbb{Z}$, one can replace the retraction in (3.3) with the Demazure operator

$$
\mathbb{Z}[\mathbf{x}] \stackrel{\pi_{w_{0}(J)}}{\longrightarrow} \mathbb{Z}[\mathbf{x}]^{W_{J}}
$$

associated to the longest element $w_{0}(J)$ in $W_{J}$, where

$$
\pi_{s_{i}}(f):=\frac{x_{i} f-x_{i+1} s_{i}(f)}{x_{i}-x_{i+1}}
$$

and $\pi_{w}:=\pi_{s_{i_{1}}} \cdots \pi_{s_{i_{\ell}}}$ if $w=s_{i_{1}} \cdots s_{i_{\ell}}$ is any reduced decomposition for $w$.

In type $A_{n-1}$, one can replace the Hiller Schubert polynomial $S_{w}$ with Lascoux and Schützenberger's Schubert polynomial $\mathfrak{S}_{w}$ (see for example [M91, M01]): one chooses the root linear functionals to be $x_{i}-x_{j}$ for $1 \leq i \neq j \leq n$ and replaces the previous choice of $S_{w_{0}}=\prod_{i<j}\left(x_{i}-x_{j}\right)$ with an element which is equivalent modulo the ideal $\left(\mathbb{Z}[\mathbf{x}]_{+}^{W}\right)$, namely

$$
\mathfrak{S}_{w_{0}}:=\mathbf{x}^{\delta_{n}}:=x_{1}^{n-1} x_{2}^{n-2} \cdots x_{n-1}^{1} x_{n}^{0} .
$$

Defining $\mathfrak{S}_{w}:=\partial_{w^{-1} w_{0}} \mathfrak{S}_{w_{0}}$, property (c) from Section 2 tells us that the images of $S_{w}$ and $\mathfrak{S}_{w}$ within $\mathbb{Z}[\mathbf{x}] /\left(\mathbb{Z}[\mathbf{x}]_{+}^{W}\right)$ are the same for all $w \in W$. These $\mathfrak{S}_{w}$ :

- lie in $\mathbb{Z}[\mathbf{x}]$ and have non-negative integer coefficients,

- lift the cohomology classes $\sigma_{w}$ in the cohomology with integer coefficients

$$
H^{\star}(G / B, \mathbb{Z}) \cong \mathbb{Z}[\mathbf{x}] /\left(\mathbb{Z}[\mathbf{x}]_{+}^{W}\right),
$$

and

- give us Schur functions in finite variable sets whenever $w$ is grassmannian: if one has $\operatorname{Des}(w) \subseteq\{(r, r+1)\}$ (in which case we say $u$ is $r$-grassmannian) so that

$$
w_{1}<w_{2}<\cdots<w_{r} \text { and } w_{r+1}<w_{r+2}<\cdots<w_{n}
$$

then

$$
\mathfrak{S}_{w}=s_{\lambda}\left(x_{1}, \ldots, x_{r}\right),
$$

where $\lambda$ is the partition $\lambda=\left(w_{r}-r, \ldots, w_{2}-2, w_{1}-1\right)$. Note that $\lambda$ has at most $r$ parts, all of size at most $n-r$, so its Young diagram fits inside an $r \times(n-r)$ rectangle.

In order to both suppress the variable set $x_{1}, \ldots, x_{r}$ from the notation and make more convenient use of symmetric function identities, we will work within a quotient of the ring of symmetric functions with integral coefficients

$$
\Lambda=\Lambda_{\mathbb{Z}}\left(x_{1}, x_{2}, \ldots\right)
$$

see [M95, Chapter 1], S99, Chapter 7]. The $\mathbb{Z}$-basis for $\Lambda$ given by the Schur functions $s_{\lambda}$ has the property that the $\mathbb{Z}$-submodule $I_{r, n-r}$ spanned by all $s_{\lambda}$ with 
$\lambda \nsubseteq(n-r)^{r}$ forms an ideal, and the map sending $s_{\lambda}$ to $s_{\lambda}\left(x_{1}, \ldots, x_{r}\right)$ induces an isomorphism

$$
\Lambda / I_{r, n-r} \stackrel{\sim}{\longrightarrow} \mathbb{Z}[\mathbf{x}]^{W_{J}} / \mathbb{Z}[\mathbf{x}]_{+}^{W} \mathbb{Z}[\mathbf{x}]^{W_{J}} \quad\left(\cong H^{\star}\left(G / P_{J}, \mathbb{Z}\right)\right) .
$$

Thus $H^{\star}\left(G / P_{J}, \mathbb{Z}\right)$ has $\mathbb{Z}$-basis given by

$$
\left\{\sigma_{w}: w \in W^{J}\right\}=\left\{s_{\lambda}: \lambda \subseteq(n-r)^{r}\right\} .
$$

4.4. A shorter presentation in type $A$. Starting with Theorem 2.3 , our goal is to find an even smaller set of generators for the ideal $J_{v_{r, s, t, n}}$ within the coinvariant algebra, so that through (1.6) we obtain an even shorter presentation of $I_{w}$ in type $A$.

First note that even though our proof of Theorem 2.3 for all finite Coxeter groups was done in $\mathbb{k}[V] /\left(\mathbb{k}[V]_{+}^{W}\right)$ where the field $\mathbb{k}$ has characteristic zero, the same proof works in type $A$ more generally for the integral coinvariant algebra $H^{\star}(G / B, \mathbb{Z})$. This follows since the Schubert polynomials $\left\{\mathfrak{S}_{w}\right\}$ satisfy the integer coefficient versions of all of the requisite properties (a)-(g) used in Section 2.

The bigrassmannian $v_{r, s, t, n}$ described explicitly in (4.3) is $r$-grassmannian and corresponds to the $j \times i$ rectangular partition $i^{j}$, where we define

$$
\begin{aligned}
& i:=s-t+1, \\
& j:=r-t+1 .
\end{aligned}
$$

When $u$ and $v$ are $r$-grassmannian and correspond respectively to partitions $\lambda$ and $\mu$, the Bruhat order relation $u \geq v$ is equivalent to the inclusion $\lambda \supseteq \mu$ of their Young diagrams, meaning that $\lambda_{i} \geq \mu_{i}$ for all $i$. Thus Theorem 2.3 says that $J_{v_{r, s, t, n}}$ is generated as an ideal of $\Lambda / I_{r, n-r}$ by

$$
\left\{\mathfrak{S}_{u}: \operatorname{Des}(u)=\{(r, r+1)\}, u \geq v_{r, s, t, n}\right\}=\left\{s_{\mu}: i^{j} \subseteq \mu \subseteq(n-r)^{r}\right\} .
$$

This presentation from Theorem 2.3 can be improved in type $A_{n-1}$ as follows:

Theorem 4.8. Given a bigrassmannian $v=v_{r, s, t, n}$ in type $A_{n-1}$ with $\mathfrak{S}_{v}=s_{i^{j}}$, let

$$
\begin{aligned}
a & :=\min (n-r-i, r-j), \\
b & :=\min (i, j) .
\end{aligned}
$$

Then $J_{v}$ is generated as an ideal of $H^{\star}\left(G / P_{J}, \mathbb{Z}\right) \cong \Lambda / I_{r, n-r}$ by

$$
\left\{s_{\mu}: i^{j} \subseteq \mu \subseteq\left((i+a)^{b}, i^{j-b}\right)\right\} .
$$

Alternatively, $J_{v}$ is generated by

$$
\left\{s_{\mu}: i^{j} \subseteq \mu \subseteq\left(i^{j}, b^{a}\right)\right\} .
$$

We delay our proof of Theorem 4.8 until Section 4.7

Note that in both of the asserted generating sets (4.5) and (4.6) for $J_{v}$, the shapes $\mu$ indexing the generators $s_{\mu}$ run through an interval between the $j \times i$ rectangular shape $i^{j}$ and the disjoint union of $i^{j}$ with a smaller rectangle of shape $a \times b$ or $b \times a$. In (4.5) the smaller rectangle is to the right of the rectangle $i^{j}$, with both top-justified, while in (4.6) the smaller rectangle is below the rectangle $i^{j}$, with both left-justified. Thus in both cases, the generating sets have size $\left(\begin{array}{c}a+b \\ a\end{array}\right)$ and consist of generators whose multiset of degrees have generating function

$$
\sum_{\mu} q^{|\mu|}=q^{i j}\left[\begin{array}{c}
a+b \\
a
\end{array}\right]_{q},
$$


where $\left[\begin{array}{c}a+b \\ a\end{array}\right]_{q}$ is a $q$-binomial coefficient or Gaussian polynomial [M91, I.2 Exer. $3]$.

Example 4.9. We examine the special case where the bigrassmannian $v:=v_{r, s, t, n}$ has $t$ equal to $r$ or $s$, so that the Schubert condition $C_{r, s, t}$ in (4.1) becomes an inclusion $V_{r} \subseteq \mathbb{C}^{s}$ or $V_{r} \supseteq \mathbb{C}^{s}$. Schubert varieties $X_{w}$ in type $A$ for which all Schubert conditions on $X_{w}$ take one of these two forms were called Schubert varieties defined by inclusions in GR02. That paper gave a presentation for the cohomology containing

- for each inclusion condition $V_{r} \supseteq \mathbb{C}^{s}$, a set of $s$ generators for $J_{v}$ of the form

$$
\left\{e_{m}\left(x_{1}, \ldots, x_{r}\right)\right\}_{m=r-s+1, r-s+2, \ldots, r},
$$

- and for each inclusion condition $V_{r} \subseteq \mathbb{C}^{s}$, a set of $r$ generators for $J_{v}$ of the form

$$
\left\{e_{m}\left(x_{r}+1, \ldots, x_{n}\right)\right\}_{m=s-r+1, s-r+2, \ldots, n-r} .
$$

We compare this with the presentation for $J_{v}$ in Theorem 4.8 say for the inclusion conditions of the form $V_{r} \supseteq \mathbb{C}^{s}$, and using the generators given in (4.6).

Since $t=s$, one has

$$
\begin{aligned}
& i=s-t+1=1, \\
& j=r-s+1, \\
& b=\min (i, j)=i=1, \\
& a=\min (n-r-1, s-1) .
\end{aligned}
$$

Hence (4.6) says that $J_{v}$ is generated by the set of $a+1$ Schur functions

$$
\left\{s_{\mu}: 1^{r-s+1} \subseteq \mu \subseteq 1^{a+r-s+1}\right\}=\left\{e_{m}\left(x_{1}, \ldots, x_{r}\right)\right\}_{m=r-s+1, r-s+2, \ldots, a+r-s+1}
$$

which is exactly the first $a+1=\min (n-r, s)$ from the $s$ generators listed in (4.7). Hence Theorem 4.8 provides a dramatic reduction in the size of the generating set for $J_{v}$ whenever $n-r$ is small compared to $s$.

We also remark that the techniques utilized in GR02 seem very particular to the case where $X_{w}$ is defined by inclusions. We do not know how to use them for some alternate approach to the case of general $X_{w}$ considered in this paper.

4.5. A minimality conjecture. As we shall see in a moment, our generators for $I_{w}$ are not minimal. However, we believe the following holds:

Conjecture 4.10. The two generating sets for the ideal $J_{v_{r, s, t, n}}$ given in Theorem 4.8 are both minimal.

Via computer, we have verified this conjecture for all bigrassmannian permutations where $r \leq 4$ and $n-r \leq 5$.

In fact, Conjecture 4.10 indicates obstructions to short presentations of $H^{\star}\left(X_{w}\right)$ in general. We now give a family of ideals that would require a large number of generators if the conjecture is true.

For a positive integer $m$, let $n=4 m$, and consider in $W=S_{n}=S_{4 m}$ the bigrassmannian $v_{r, s, t, n}$ that corresponds to $r=n-r=2 m$ and $i=j=m$. Then 
$a=b=m$, and $J_{v_{r, s, t, n}}\left(=I_{w_{r, s, t, n}}\right)$ requires

$$
\left(\begin{array}{c}
2 m \\
m
\end{array}\right) \sim \frac{4^{m}}{\sqrt{\pi m}}=\frac{\sqrt{2}^{n+2}}{\sqrt{\pi n}}
$$

generators according to Conjecture 4.10 .

The size of any minimal generating set of a homogeneous ideal is well defined. This is implied by the following well-known fact:

Proposition 4.11. Let $R$ be a commutative ring, and $\Lambda=\bigoplus_{n \geq 0} \Lambda_{n}$ a graded, connected $R$-algebra, meaning that $\Lambda_{0}=R$ and $\Lambda_{i} \Lambda_{j} \subset \Lambda_{i+j}$. Let $M$ be a graded $\Lambda$-module, with degrees bounded below, meaning that $M=\bigoplus_{n \geq N} M_{n}$ for some $N \in \mathbb{Z}$, and $\Lambda_{i} M_{j} \subset M_{i+j}$.

Then a set of homogeneous elements $\left\{m_{i}\right\}_{i=1}^{t}$ generate $M$ as an $\Lambda$-module if and only if their images $\left\{\bar{m}_{i}\right\}_{i=1}^{t}$ span $M / \Lambda_{+} M$ as an $R$-module. In particular, $\left\{m_{i}\right\}_{i=1}^{t}$ form a minimal $\Lambda$-generating set with respect to inclusion for $M$ if and only if $\left\{\bar{m}_{i}\right\}_{i=1}^{t}$ form a minimal $R$-spanning set for $M / \Lambda_{+} M$.

In our setting, the well-definedness follows by setting $\Lambda=\bigoplus_{n \geq 0} \Lambda_{n}$ to be the graded ring of symmetric functions with $\mathbb{Z}$ coefficients and by setting $M=J_{v_{r, s, t, n}}$, so that the $\mathbb{Z}$-module $M / \Lambda_{+} M$ is a finitely generated abelian group. Thus we conjecture that this abelian group $M / \Lambda_{+} M$ requires $\left(\begin{array}{c}a+b \\ a\end{array}\right)$ generators, and, in fact,

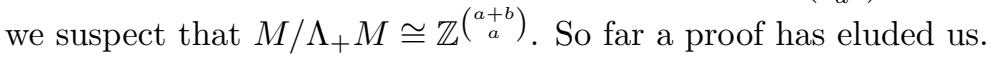

Example 4.12. Since $I_{w}=\sum_{v \in \mathcal{E}(w)} J_{v}$, and since we have conjectured that the generating sets provided by Theorem 4.8 for $J_{v}$ are minimal, one might wonder whether their concatentation gives a minimal generating set of $I_{w}$. As mentioned above, this turns out to be false in general.

The smallest counterexample is given by $w=1243$, which has

$$
\mathcal{E}(w)=\left\{v_{1}=2134, v_{2}=1324\right\} .
$$

The generating sets given in Theorem 4.8 for the ideals $J_{v_{1}}$ and $J_{v_{2}}$ are

$$
\begin{aligned}
J_{v_{1}} & =\left\langle s_{(1)}\left(x_{1}\right)\right\rangle=\left\langle x_{1}\right\rangle, \\
J_{v_{2}} & =\left\langle s_{(1)}\left(x_{1}, x_{2}\right), \quad s_{(2)}\left(x_{1}, x_{2}\right)\right\rangle \quad \text { or } \quad\left\langle s_{(1)}\left(x_{1}, x_{2}\right), \quad s_{(1,1)}\left(x_{1}, x_{2}\right)\right\rangle \\
& =\left\langle x_{1}+x_{2}, \quad x_{1}^{2}+x_{1} x_{2}+x_{2}^{2}\right\rangle \quad \text { or } \quad\left\langle x_{1}+x_{2}, \quad x_{1} x_{2}\right\rangle,
\end{aligned}
$$

and in each case they minimally generate their ideals $J_{v_{i}}$. However, concatenating them gives non-minimal generating sets for $I_{w}$, namely

$$
\begin{aligned}
I_{w}=J_{v_{1}}+J_{v_{2}} & =\left\langle x_{1}, \quad x_{1}+x_{2}, \quad x_{1}^{2}+x_{1} x_{2}+x_{2}^{2}\right\rangle \quad \text { or } \quad\left\langle x_{1}, \quad x_{1}+x_{2}, \quad x_{1} x_{2}\right\rangle \\
& \left(=\left\langle x_{1}, \quad x_{1}+x_{2}\right\rangle\right) .
\end{aligned}
$$

Example 4.13. Some readers may find the above earliest example artificial: although $X_{w}=X_{1243}$ lives inside $G L_{4} / B$, it is isomorphic to $X_{21}=G L_{2} / B \cong \mathbb{P}^{2}$. However, one can easily produce from this more counterexamples with similar properties but with no such artificial nature.

For example, take $w=23541$, which has $\mathcal{E}(w)=\left\{v_{1}=31245, v_{2}=14235\right\}$. Then $J_{v_{1}}$ and $J_{v_{2}}$ require one and two generators, respectively, but the sum $I_{w}=J_{v_{1}}+J_{v_{2}}$ requires only two generators, not three. 
4.6. Some symmetric function identities. The proof of Theorem 4.8 on generators for $J_{v_{r, s, t, n}}$ will use some symmetric function identities which we describe and prove in this section. We will make use of standard terminology, such as in [M95, S01, S99].

In particular, we will use the Pieri rule expanding the product of an elementary symmetric function $e_{r}:=s_{1^{r}}$ with an arbitrary Schur function into Schur functions:

$$
e_{k} s_{\lambda}=\sum_{\mu} s_{\mu},
$$

where the sum runs over all partitions $\mu$ obtained from $\lambda$ by adding on a vertical strip of length $k$. The following easy consequence will be used in the proof of Theorem 4.8 below.

Lemma 4.14. For any partition $\nu$ and non-negative integer $k$, one has

$$
s_{\left(\nu, 1^{k}\right)}=\sum_{\ell=0}^{k}(-1)^{\ell} e_{k-\ell} \sum_{\lambda} s_{\lambda},
$$

where the inner sum runs over partitions $\lambda$ obtained from $\nu$ by adding a horizontal strip of length $\ell$.

Proof. Using the Pieri rule (4.10) to expand the right side of (4.11), one obtains

$$
\sum_{\ell=0}^{k}(-1)^{\ell} e_{k-\ell} \sum_{\lambda} s_{\lambda}=\sum_{(\ell, \lambda)}(-1)^{\ell} s_{\lambda},
$$

where the sum runs over pairs $(\ell, \lambda)$ in which both $0 \leq \ell \leq k$ and $\lambda$ is obtained from $\nu$ by first adding a horizontal $\ell$-strip within the first $\ell(\nu)$ rows and then adding an arbitrary vertical $(k-\ell)$-strip. Cancel all these pairs, except for the one with $\ell=0$ and $\lambda=\left(\nu, 1^{k}\right)$, via the following sign-reversing involution: if $x$ (respectively $y$ ) is the farthest east (respectively, farthest north) box in the horizontal (respectively vertical) strip, then

- when $y$ is to the right of $x$ (or when $\ell=0$ and $\lambda \neq\left(\nu, 1^{k}\right)$ ), move $y$ from the vertical to the horizontal strip, and,

- when $y$ is below $x$, move $x$ from the horizontal strip to the vertical strip.

We also need the Jacobi-Trudi identity:

$$
s_{\lambda}=\operatorname{det}\left(h_{\lambda_{i}-i+j}\right)_{i, j=1,2, \ldots, \ell(\lambda)}
$$

with the usual convention that $h_{r}:=s_{(r)}$ for $r \geq 0$ and $h_{r}=0$ for $r<0$. This has the following consequence, also to be used in the proof of Theorem 4.8 below.

Lemma 4.15. Let $i<k$, and assume $\mu$ is a partition with $\mu_{k}>i \geq \mu_{k+1}$, so that the $(i+1)^{\text {st }}$ column of the Young diagram for $\mu$ has length $k>i$. Then

$$
s_{\mu}=\sum_{m=1}^{k}(-1)^{k-m} h_{\mu_{m}+k-i-m} s_{\mu^{(m)}},
$$

where for $m=1,2, \ldots, k$ one defines

$\mu^{(m)}:=\left(\mu_{1}, \mu_{2}, \ldots, \mu_{m-1}, \widehat{\mu_{m}}, \mu_{m+1}-1, \mu_{m+2}-1, \ldots, \mu_{k}-1, i, \mu_{k+1}, \mu_{k+2}, \ldots, \mu_{\ell}\right)$, where $\ell:=\ell(\mu)$ and $\widehat{\mu_{m}}$ refers to the deletion of the entry $\mu_{m}$. 
Proof. Start with the $\ell \times \ell$ Jacobi-Trudi matrix for $\mu$. From this create an $(\ell+1) \times \ell$ matrix by inserting a new row between its row $k$ and row $k+1$, having entries

$$
\left(h_{i-k+1}, h_{i-k+2}, \ldots, h_{i-k+\ell}\right) \text {. }
$$

Then from this $(\ell+1) \times \ell$ matrix, create a singular $(\ell+1) \times(\ell+1)$ matrix by introducing an $(\ell+1)^{s t}$ column that duplicates the $(k-i)^{t h}$ column. This last duplicated column is

$$
\begin{aligned}
& \left(h_{\mu_{1}+k-i-1}, \ldots, h_{\mu_{k}-i}, \quad h_{i-k+(k-i)}, \quad h_{\mu_{k+1}-i-1}, \quad \ldots, \quad h_{\mu_{\ell}+k-i-\ell}\right)^{T} \\
& =\left(\begin{array}{ccccc}
h_{\mu_{1}+k-i-1}, \ldots, h_{\mu_{k}-i}, & 1, & 0, & \ldots, & 0
\end{array}\right)^{T} .
\end{aligned}
$$

Here we have used the fact that $h_{0}=1$ and that $h_{\mu_{m}+k-i-m}=0$ for $m \geq k+1$ because $\mu_{m} \leq \mu_{k+1} \leq i$ implies $\mu_{m}+k-i-m=\left(\mu_{m}-i\right)+(k-m)<0$. One then checks that the cofactor expanding the (zero) determinant of this $(\ell+1) \times(\ell+1)$ matrix along this duplicated column gives the asserted identity.

4.7. Proof of Theorem 4.8. The proof of the second statement will follow from the first, via the well-known ring involution $\omega$ on symmmetric functions defined by

$$
\begin{aligned}
& \Lambda \stackrel{\omega}{\rightarrow} \Lambda, \\
& s_{\lambda} \longmapsto s_{\lambda^{\prime}},
\end{aligned}
$$

where $\lambda^{\prime}$ is the conjugate partition to $\lambda$. This means that $\omega$ sends the ideal $I_{r, n-r}$ to the ideal $I_{n-r, r}$. Hence the set (4.6) generates $J_{v}$ within $\Lambda / I_{r, n-r}$, where $v$ corresponds to an $i \times j$ rectangle, if and only if the set (4.5) generates the ideal $J_{v^{\prime}}$ within $\Lambda / I_{n-r, r}$, where $v^{\prime}$ corresponds to a $j \times i$ rectangle.

The proof for (4.5) is by induction on the degree $d$, which is the number of boxes in our partition. Our inductive hypothesis is that the portion of $J_{v}$ of degree at most $d$ is generated by those elements of (4.5) of degree at most $d$, or equivalently, that all elements of (4.4) of degree at most $d$ are writable in terms of elements of (4.5) of degree at most $d$. The base case, $d=i j$, is clear, since $s_{i^{j}}$ is the only element of degree $i j$ in both sets.

Our proof for the inductive case proceeds in three steps. Start with the generating set for $J_{v}$ given in (4.4). We wish to show that, modulo $I_{r, n-r}$, all such $s_{\mu}$ with $|\mu|=d$ lie in the ideal generated by those $s_{\mu}$ with $|\mu|<d$ and those

Step 1 . with $\mu$ in the interval $\left[i^{j},(n-r)^{j}\right]$, and then furthermore

Step 2. with $\mu$ in the interval $\left[i^{j},(i+a)^{j}\right]$, and then finally

Step 3. with $\mu$ in the interval $\left[i^{j},\left((i+a)^{b}, i^{j-b}\right)\right]$.

Step 1. We will use induction on a certain partial order on partitions which depends on the index $j$. For a partition $\lambda$, define

$$
\hat{\lambda}:=\left(\lambda_{j+1}, \lambda_{j+2}, \ldots\right),
$$

so that the Young diagram of $\hat{\lambda}$ consists of rows $j+1, j+2, \ldots$ from the Young diagram of $\lambda$. Then partially order the partitions containing $i^{j}$ by decreeing $\lambda \prec_{j} \mu$ if either $|\hat{\lambda}|<|\hat{\mu}|$, or if $|\hat{\lambda}|=|\hat{\mu}|$ but $\hat{\lambda}<\hat{\mu}$ in the dominance order, meaning that

$$
\lambda_{j+1}+\lambda_{j+2}+\cdots \lambda_{k} \leq \mu_{j+1}+\mu_{j+2}+\cdots \mu_{k}
$$

for each $k \geq j$.

Now if $\mu$ does not already lie in the interval $\left[i^{j},(n-r)^{j}\right]$, so that $\ell(\mu)=k+j>j$, let $\nu$ be the partition obtained from $\mu$ by removing 1 from its last $k$ non-empty 
parts $\mu_{j+1}, \mu_{j+2}, \ldots, \mu_{j+k}$. Then by the Pieri rule (4.10), $e_{k} s_{\nu}=s_{\mu}+\sum_{\lambda} s_{\lambda}$, where $\lambda$ runs through partitions other than $\mu$ obtained from $\nu$ by adding a vertical strip of size $k$. One can check that any such $\lambda$ satisfies $\lambda \prec_{j} \mu$ : either the vertical strip contains some boxes in the first $j$ rows, so that $|\hat{\lambda}|<|\hat{\mu}|$, or if not, the location of the vertical strip forces $\hat{\lambda}<\hat{\mu}$ in dominance. Also, $k \geq 1$, so $|\nu|<|\mu|$. Consequently, by induction on the order $\prec$, one has an expression for $s_{\mu}$ showing that it is in the ideal generated by $s_{\lambda}$, where either $|\lambda|<|\mu|$ or $\lambda$ is in the interval $\left[i^{j},(n-r)^{j}\right]$.

Step 2. We will again use induction, this time on reverse dominance order. We wish to write $s_{\mu}$, where $\mu$ is in the interval $\left[i^{j},(n-r)^{j}\right]$ in terms of $s_{\lambda}$, where $|\lambda|<|\mu|$ or $\lambda$ lies in the interval $\left[i^{j},(i+a)^{j}\right]$. Recall that $a=\min (n-r-i, r-j)$, and if $a=n-r-i$, then $n-r=i+a$, so there is nothing to do after Step 1. Thus we may assume $a=r-j$.

If $\mu$ does not already lie in the interval $\left[i^{j},(i+a)^{j}\right]$, so that $\mu_{1}>i+a$, let $k:=\mu_{1}-i>a$, and let $\nu$ be the partition obtained from $\mu$ by removing one box from each of the last $k$ non-empty columns in the Young diagram of $\mu$. Note that $\ell(\nu) \geq \ell(\mu)$ since $k<\mu_{1}$ and that $\ell(\mu) \geq j$ since $i^{j} \subset \mu$. Hence $\left(\nu, 1^{k}\right)$ has length at least $j+k>j+a=r$, so that the partition $\left(\nu, 1^{k}\right) \nsubseteq(n-r)^{r}$, and hence Lemma 4.14 tells us that

$$
\sum_{\ell=0}^{k}(-1)^{\ell} e_{k-\ell} \sum_{\lambda} s_{\lambda} \equiv 0 \quad \bmod I_{r, n-r}
$$

where in the sum $\lambda$ runs through partitions having no more than $j$ parts obtained from $\nu$ by adding a horizontal strip of length $\ell$.

We claim that almost all of the terms in this sum, except the single term with $\ell=k$ and $\lambda=\mu$, will have $|\lambda|<|\mu|$ or $\lambda>\mu$. If $\ell<k$, then $|\lambda|<|\mu|$. If $\ell=k$, note the horizontal strip $\lambda / \nu$ cannot have any boxes in the first $i$ columns, as those already have length $j$ in $\nu$. Therefore, the location of the horizontal strip forces $\lambda>\mu$ in dominance. Consequently, by induction, one has an expression for $s_{\mu}$ showing that it is in the ideal generated by $s_{\lambda}$, where $|\lambda|<|\mu|$ or $\lambda$ is in the interval $\left[i^{j},(i+a)^{j}\right]$.

Step 3. Now we show that, if $\mu$ fits inside $(i+a)^{j}$ but not inside $\left((i+a)^{b}, i^{j-b}\right)$, then $s_{\mu}$ can always be written as a sum of terms of the form $h_{r} s_{\lambda}$ for $r>0$ and $\lambda$ containing $i^{j}$. Since $r>0,|\lambda|<|\mu|$, this suffices to finish the proof.

Recall that $b=\min (i, j)$, and if $b=j$, then there is nothing to do after Step 2. Thus we may assume $b=i<j$.

Let $k$ be the number of parts of $\mu$ which are strictly larger than $i$, so that $k$ is the size of the $(i+1)^{\text {st }}$ column in the Young diagram of $\mu$. Since $\mu$ does not fit inside $\left((i+a)^{i}, i^{j-i}\right)$, it must be that $k>i$, and we are in the situation of Lemma 4.15. Hence

$$
s_{\mu}=\sum_{m=1}^{k}(-1)^{k-m} h_{\mu_{m}+k-i-m} s_{\mu^{(m)}},
$$

where

$$
\mu^{(m)}:=\left(\mu_{1}, \mu_{2}, \ldots, \mu_{m-1}, \mu_{m+1}-1, \mu_{m+2}-1, \ldots, \mu_{k}-1, i, \mu_{k+1}, \mu_{k+2}, \ldots, \mu_{\ell}\right)
$$

and $\ell=\ell(\mu)$. Note that, since $\mu$ contains $i^{j}$, and hence $k \leq j$, each $\mu^{(m)}$ also contains $i^{j}$. Also note that each factor $h_{\mu_{m}+k-i-m}$ has positive degree: $m \leq k$ implies $\mu_{m} \geq \mu_{k}>i$, and hence $\mu_{m}+k-i-m=\left(\mu_{m}-i\right)+(k-m)>0$. 


\section{A QUeSTION}

Question 5.1. Can one find a minimal generating set for the ideal $I_{w}$ in type $A_{n-1}$ ?

Can this at least be done for some of the recently-studied subclasses GR02, HLSS07, OPY07, where $I_{w}$ can be generated by $n^{2}$ elements, such as

- when $X_{w}$ is defined by inclusions, which occurs when $w$ avoids the patterns

$$
\{4231,35142,42513,351624\} \text {, }
$$

- or more restrictively, when $X_{w}$ is smooth, which occurs when $w$ avoids the patterns

$$
\{3412,4231\} ?
$$

It was mentioned in the Introduction that for a special subclass of smooth Schubert varieties $X_{w}$ originally considered by Ding D97, D01, there is a known minimal (in fact, complete intersection) presentation for $H^{*}\left(X_{w}, \mathbb{Z}\right)$ with $n$ relations that was exploited in DMR07. Short presentations would be useful to extend that work further.

\section{ACKNOWLEDGEMENTS}

The authors thank Nathan Reading for helpful comments and corrections. We also thank the anonymous referee for pointing out the reference ALP92 to us, and for other helpful remarks. The first author was supported by NSF grant DMS0601010. The third author was supported by NSF grants DMS-0601010 and DMS0901331. The second author was supported by NSF VIGRE grant DMS-0135345. This work was partially completed while the third author was a visitor at the Fields Institute in Toronto, and was facilitated by a printer graciously provided by Lawrence Gray through the University of Minnesota.

\section{REFERENCES}

[ALP92] E. Akyildiz, A. Lascoux and P. Pragacz, Cohomology of Schubert subvarieties of $G L_{n} /$ P. J. Differential Geometry 35 (1992), 511-519. MR.1163446 (93g:14058)

[BGG73] I.N. Bernstein, I.M. Gel'fand, S.I. Gel'fand, Schubert cells, and the cohomology of the spaces G/P. Uspehi Mat. Nauk. 28 (1973), no. 3(171), 3-26 [Russian Math. Surveys 28 (1973), no. 3, 1-26]. MR0429933 (55:2941)

[BL00] S. Billey and V. Lakshmibai, Singular loci of Schubert varieties. Progr. Math. 182, Birkhäuser, Boston, 2000. MR1782635 (2001j:14065)

[BB05] A. Björner and F. Brenti, Combinatorics of Coxeter groups. Graduate Texts in Mathematics 231, Springer-Verlag, New York, 2005. MR2133266 (2006d:05001)

[B53] A. Borel, Sur la cohomologie des espaces fibrés principaux et des espaces homogènes de groupes de Lie compacts. Ann. of Math. 57 (1953), 115-207. MR0051508(14:490e)

[C92] J.B. Carrell, Some remarks on regular Weyl group orbits and the cohomology of Schubert varieties, in Kazhdan-Lusztig theory and related topics (Chicago, IL, 1989), 33-41. Contemp. Math. 139, Amer. Math. Soc., Providence, RI, 1992. MR.1197828(93k:14068)

[D73] M. Demazure, Invariants symétriques entiers des groupes de Weyl et torsion. Invent. Math. 21 (1973), 287-301. MR0342522 (49:7268)

[DMR07] M. Develin, J. Martin, V. Reiner, Classification of Ding's Schubert varieties: Finer rook equivalence. Canad. J. Math. 59 (2007), no. 1, 36-62. MR2289417(2008b:14080)

[D97] K. Ding, Rook placements and cellular decomposition of partition varieties. Discrete Math. 170 (1997), no. 1-3, 107-151. MR1452940(98i:05166)

[D01] Rook placements and classification of partition varieties $B \backslash M_{\lambda}$. Commun. Contemp. Math. 3 (2001), no. 4, 495-500. MR1869101 (2003a:05154) 
[EL96] K. Eriksson and S. Linusson, Combinatorics of Fulton's essential set, Duke Math. J. 85 (1996), 61-76. MR1412437 (98b:05105)

[F92] W. Fulton, Flags, Schubert polynomials, degeneracy loci, and determinantal formulas. Duke Math. J. 65 (1992), no. 3, 381-420. MR.1154177 (93e:14007)

[FP98] W. Fulton and P. Pragacz, Schubert varieties and degeneracy loci. Lecture Notes in Mathematics 1689, Springer-Verlag, Berlin, 1998. MR1639468 (99m:14092)

[GR02] V. Gasharov and V. Reiner, Cohomology of smooth Schubert varieties in partial flag manifolds. J. London Math. Soc. 66 (2002), no. 3, 550-562. MR 1934291 (2003i:14064)

[GK97] M. Geck and S. Kim, Bases for the Bruhat-Chevalley order on all finite Coxeter groups. J. Algebra 197 (1997), no. 1, 278-310. MR.1480786 (98k:20066)

[H82] H. Hiller, Geometry of Coxeter groups. Research Notes in Mathematics 54, Pitman (Advanced Publishing Program), Boston-London, 1982. MR649068 (83h:14045)

[HLSS07] A. Hultman, S. Linusson, J. Shareshian, and J. Sjöstrand, From Bruhat intervals to intersection lattices and a conjecture of Postnikov. J. Combin. Theory Ser. A 116 (2009), no. 3, 564-580. MR2500158

[H90] J. Humphreys, Reflection groups and Coxeter groups. Cambridge Studies in Advanced Mathematics 29, Cambridge University Press, Cambridge, 1990. MR 1066460 (92h:20002)

[LS96] A. Lascoux and M.-P. Schützenberger, Treillis et bases des groupes de Coxeter. Electron. J. Combin. 3 (1996), no. 2, R27, 35 pp. (electronic). MR1395667 (98c:05168)

[M95] I.G. Macdonald, Symmetric functions and Hall polynomials. Second edition. Oxford Mathematical Monographs. Oxford University Press, New York, 1995. MR.1354144 (96h:05207)

[M91] Notes on Schubert polynomials, Publications du LaCIM. Université du Québec à Montréal, 1991.

[M01] L. Manivel, Symmetric functions, Schubert polynomials and degeneracy loci. $S M F / A M S$ Texts and Monographs 6, American Mathematical Society, Providence, RI, 2001. MR1852463 (2002h:05161)

[OPY07] S. Oh, A. Postnikov, and H. Yoo Bruhat order, smooth Schubert varieties, and hyperplane arrangements. J. Combin. Theory Ser. A 115 (2008), no. 7, 1156-1166. MR2450335

[R02] N. Reading, Order dimension, strong Bruhat order and lattice properties for posets. Order 19 (2002), no. 1, 73-100. MR1902662 (2003m:05214)

[S01] B.E. Sagan, The symmetric group: Representations, combinatorial algorithms, and symmetric functions, 2nd ed. Graduate Texts in Mathematics 203, Springer-Verlag, New York, 2001. MR1824028 (2001m:05261)

[S99] R.P. Stanley, Enumerative Combinatorics, Volume 2. Cambridge Studies in Advanced Mathematics 62, Cambridge University Press, Cambridge, 1999. MR:1676282 (2000k:05026)

School of Mathematics, University of Minnesota, Minneapolis, Minnesota 55455

E-mail address: reiner@math.umn.edu

Department of Mathematics, Statistics, and Computer Science, Saint Olaf College, Northfield, Minnesota 55057

E-mail address: woo@stolaf.edu

Department of Mathematics, University of Illinois at Urbana-Champaign, Urbana, ILLINOIS 61801

E-mail address: ayong@illinois.edu 